\title{
Surveillance System Evaluations Provide Evidence to Improve Public Health Practice
}

\author{
Beverley J. Paterson*1 and David N. Durrheim²,1 \\ ${ }^{1}$ Hunter Medical Research Unit, University of Newcastle, Newcastle, NSW, Australia; ${ }^{2 H}$ Hunter New England Population Health, \\ Newcastle, NSW, Australia
}

\section{Objective}

Surveillance evaluations should not only describe surveillance systems but provide evidence to improve public health practice. This presentation documents how knowledge gathered through a syndromic surveillance evaluation in Pacific Island Countries and Territories (PICTs) with local health personnel was translated into action, in collaboration with global health partners.

\section{Introduction}

A simplified syndromic surveillance system, based on early detection and reporting of four core syndromes and immediate reporting of unusual events, was established across PICTs in 2010. An early evaluation of the system was undertaken to make recommendations on improvements(1). The evaluation examined whether the system was meeting its objective of serving as an early warning system and its capacity to investigate and respond to outbreaks. Metrics included system acceptability, data quality, timeliness and level of compliance. The evaluation identified a critical need to better equip local public health officials with the knowledge and skills to rapidly and appropriately respond to suspected infectious disease outbreaks across the Pacific.

In response to the evaluation findings, the RAPID (Response and Analysis for Pacific Infectious Diseases) project was implemented to strengthen capacity in surveillance, epidemiology and outbreak response across the PICTs. Principally funded by Australian aid and developed in partnership with the World Health Organization (WHO), the Secretariat of the Pacific Community (SPC) and the Pacific Public Health Surveillance Network (PPHSN), RAPID is an example of a multi-organisational approach to swiftly address identified surveillance issues and strengthen regional surveillance capacity.

\section{Methods}

Following identification of critical surveillance needs, implementation of the RAPID project involved public health epidemiologists and physicians facilitating core project components across the PICTs. The core components were as follows:

Component 1: Improved understanding and skills among PICT syndromic surveillance focal points to ensure strengthened detection, investigation and response to outbreaks in PICTs

-Sub-regional/national structured training workshops

-Pacific Outbreak Manual

Component 2: Strengthened institutional capacity of the Pacific International Health Regulations National Focal Points and National Outbreak Response (EpiNet) Teams

On-site capacity building, review and lessons learned training

-Mentoring

-Work exchanges

-Outbreak support

\section{Results}

The RAPID partners have cooperatively developed and conducted training programs, mentored local students, and provided in-country outbreak support. In partnership with FNU and PPHSN, an earlier course 'Data for decision-making (DDM)' was revitalised as an accredited training programme for the Pacific. In the first year, over 150 participants from 13 countries have been trained in 'Outbreak surveillance and response' and 'Basic applied epidemiology and data analysis'. The Centers for Disease Control and Prevention and the Pacific Island Health Officers' Association, partners in the PPHSN network, have collaboratively contributed to the successful running of the DDM course modules, further fostering strong linkages between the key public health organisations across the Pacific.

$R A P I D$ facilitators, working collaboratively with WHO, CDC, and local Ministry of Health officials, have also engaged with the Papua New Guinea (PNG) Field Epidemiology Training program, offering training and mentoring to PNG students. Through the RAPID project, the WHO and the Fijian Ministry of Health, were supported to rapidly establish and implement a Fijian Dengue-Like-Illness surveillance system during a major dengue outbreak. The Pacific Outbreak Manual was also further developed as an infectious disease training manual and a guide for appropriate outbreak response (http://www.spc.int/ phs/pphsn/Publications/Pacific_Outbreak_Manual_FEB2014.pdf).

\section{Conclusions}

The RAPID project is a notable example of how evidence gathered through a surveillance evaluation can be used to improve public health surveillance practice. The project showcases how gains in surveillance capacity in lower and middle income countries can rapidly be achieved through cooperative partnerships and flexible approaches.

\section{Keywords}

Surveillance; Training; Outbreak response; Capacity strengthening

\section{Acknowledgments}

The authors would like to acknowledge RAPID partners and all facilitators who have provided so much inspiration and training across the Pacific.

\section{References}

1. Paterson BJ, Kool JL, Durrheim DN, Pavlin B. Sustaining surveillance: evaluating syndromic surveillance in the Pacific. Glob Public Health 2012;7(7):682-94.

*Beverley J. Paterson

E-mail: beverley.paterson@hnehealth.nsw.gov.au and reproduction in any medium, provided the original work is properly cited. 